\title{
Circular RNA ATXN7 is upregulated in non-small cell lung cancer and promotes disease progression
}

\author{
QI HUANG ${ }^{1}$, SHAODONG WANG ${ }^{1}$, XIAO LI ${ }^{1}$, FAN YANG $^{1}$, CHANGJIANG FENG ${ }^{2}$, \\ KAIZE ZHONG $^{1}$, MANTANG QIU ${ }^{1}$ and JUN WANG ${ }^{1}$ \\ ${ }^{1}$ Department of Thoracic Surgery, Peking University People's Hospital, Beijing 100044; \\ ${ }^{2}$ Department of Thoracic Surgery, The Chinese People's Liberation Army General Hospital, Beijing 100853, P.R. China
}

Received February 8, 2018; Accepted January 24, 2019

DOI: $10.3892 / 01.2019 .10168$

\begin{abstract}
Circular RNAs (circRNAs) are a class of endogenous non-coding RNAs that is generated from back-splicing, and is characterized by a covalent closed loop without $3^{\prime}$ and 5 ' ends. Recently, the biological function of circRNAs has received increasing attention; however, studies on circRNAs in non-small cell lung cancer (NSCLC) have rarely been reported. In the present study, the expression profiles of circRNAs in NSCLC were investigated, and the association between the circular RNA ATXN7 (circATXN7) expression level and clinicopathological characteristics of patients with NSCLC was assessed. In addition, the effects of circATXN7 on cell proliferation and invasion were examined. The results revealed that circATXN7 was upregulated in 45 NSCLC tissues compared with its expression in non-tumor tissues. However, there was no marked difference between the expression level of circATXN7 and the majority of the examined clinicopathological characteristics. It was also observed that the survival time of patients with high circATXN7 levels was shorter compared with that of patients with low circATXN7 levels, although the difference was not statistically significant $(\mathrm{P}>0.05)$. Furthermore, silencing of circATXN7 by small interfering RNA inhibited the proliferation and invasion of NSCLC cells in vitro. Taken together, the present study was the first to identify that circATXN7 was upregulated in NSCLC tumor tissues. Furthermore, the downregulation of circATXN7 markedly inhibited the proliferation and invasion abilities of NSCLC cells.
\end{abstract}

Correspondence to: Dr Jun Wang or Dr Mantang Qiu, Department of Thoracic Surgery, Peking University People's Hospital, 11 South Xizhimen, Beijing 100044, P.R. China

E-mail: wangjunpkuph@sina.com

E-mail: qiumantang@163.com

Key words: circular RNA, circATXN7, non-small cell lung cancer, proliferation, prognosis

\section{Introduction}

Lung cancer is the most common malignancy and the leading cause of mortality among cancer-associated diseases worldwide (1). Non-small cell lung cancer (NSCLC) accounts for $>85 \%$ of lung cancer cases (2). The pathologic types of NSCLC include large-cell carcinoma, squamous cell carcinoma and adenocarcinoma (3). In spite of recent advances in surgical resection, chemotherapy and radiotherapy for lung cancer, the survival rate of patients remains unsatisfactory, with a 5 -year survival rate as low as $15 \%$, as it is often diagnosed at an advanced stage $(4,5)$. Therefore, there is an urgent need to identify effective molecular diagnostic markers for early detection of lung cancer (6).

Circular RNAs (circRNAs) are a class of endogenous non-coding RNAs generated from back-splicing and characterized by a covalent closed loop without $3^{\prime}$ and $5^{\prime}$ ends (7). In the past two decades, circRNAs have been hypothesized to be non-functional due to errors in splicing (8). In 2011, Salmena et al (9) presented the competing endogenous RNA hypothesis, which suggests that mRNAs, transcribed pseudogenes and long non-coding RNAs can suppress microRNA (miRNA) expression and function by competitive binding to miRNA response elements. Furthermore, it was demonstrated that non-coding RNAs are involved in the initiation and progression of malignant tumors (10). As another class of non-coding RNAs, circRNAs are more stable than linear RNAs due to their covalently closed structures, and the sponge effect of circRNAs may be more efficient in comparison with that of linear RNAs (11). Recently, a number of circRNAs have been demonstrated to regulate the function of miRNAs, serving as miRNA sponges, as well as be involved in post-transcriptional regulation in cancer (12). For instance, Zhong et al (13) reported that circTCF25 was able to downregulate miR-103a-3p and miR-107, increase cyclin-dependent kinase- 6 expression, and promote the proliferation and migration of bladder cancer cells. However, as an emerging type of non-coding RNAs, the role of circRNAs in NSCLC has rarely been reported (14).

Our previous study investigated circRNA expression in NSCLC by ribosomal RNA depletion and RNA sequencing (15). Therefore, the present study was designed to analyze the expression profile of circATXN7 in NSCLC and 
examine its correlation with clinicopathological characteristics. In addition, the effect of circATXN7 on NSCLC cell proliferation and invasion was investigated.

\section{Materials and methods}

Patients and tissue samples. A total of 57 pairs of NSCLC tissues and adjacent normal tissues ( $\geq 3 \mathrm{~cm}$ away from the tumor) were obtained from patients who underwent surgery at the Department of Thoracic Surgery, Peking University People's Hospital (Beijing, China) between January 2012 and December 2013. These tissues were used for validation of circRNAs by reverse transcription-quantitative polymerase chain reaction (RT-qPCR). All surgical specimens were snap-frozen and stored in liquid nitrogen immediately following resection until the extraction of total RNA. The clinical and pathological characteristics of each patient were collected. The Tumor-Node-Metastasis (TNM) stage was determined according to The 8th Edition Lung Cancer Stage Classification (16). The present study was approved and supervised by the Ethics Committee of Peking University People's Hospital. Written informed consent was obtained from all the patients for research purposes.

Cell culture and small interfering RNA (siRNA) transfection. The NSCLC cell lines A549 and SPC-A1 were kindly provided by Professor Xu (Jiangsu Key Laboratory of Molecular and Translational Cancer Research, Nanjing Medical University Affiliated Cancer Hospital, Nanjing, China). A549 cells were cultured in RPMI-1640 medium (Gibco; Thermo Fisher Scientific, Inc., Waltham, MA, USA), while SPC-A1 cells were grown in Dulbecco's modified Eagle's medium (DMEM; Gibco; Thermo Fisher Scientific, Inc.) supplemented with $10 \%$ fetal bovine serum (FBS; Thermo Fisher Scientific, Inc.), $100 \mathrm{U} / \mathrm{ml}$ penicillin and $100 \mu \mathrm{g} / \mathrm{ml}$ streptomycin. All cells were cultured at $37^{\circ} \mathrm{C}$ in a humidified atmosphere with $5 \% \mathrm{CO}_{2}$. For siRNA transfection, NSCLC cells were seeded in 6-well plates $\left(6 \times 10^{4}\right.$ cells/well) and cultured at $37^{\circ} \mathrm{C}$ until the cell confluence reached $60-70 \%$. Subsequently, cells were transfected with specific siRNA (100 nM) or control siRNA $(100 \mathrm{nM})$ using Lipofectamine ${ }^{\circledast}$ RNAi MAX according to the manufacturer's protocol (Invitrogen; Thermo Fisher Scientific, Inc.). Subsequent experiments were performed $24 \mathrm{~h}$ after transfection. The following siRNA sequences were used: siRNA-1 for circATXN7 sense, 5'-GGAAGGGAGCGGAAA GAAUGU-3', and antisense, 5'-AUUCUUUCCGCUCCC UUCCCG-3'; siRNA-2 for circATXN7 sense, 5'-GGGAAG GGAGCGGAAAGAAUG-3', and antisense, 5'-UUCUUU CCGCUCCCUUCCCGA-3'.

$R N A$ extraction and $R T-q P C R$ analyses. Total RNA was extracted from the tumor tissues, adjacent normal tissues and cultured cells using TRIzol reagent (Invitrogen; Thermo Fisher Scientific, Inc.) in accordance with the manufacturer's protocol. Next, the RNA concentration was measured by Uv-vis spectrophotometry (Evolution 60S; Thermo Fisher Scientific, Inc.) and 1,000 ng total RNA was reverse transcribed in a final volume of $20 \mu \mathrm{l}$ using random primers under standard conditions with the PrimeScript RT Reagent kit with gDNA Eraser (cat. no. RR047A; Takara Biotechnology Co.,
Ltd., Dalian, China). qPCR was then performed using SYBR Select Master Mix (cat. no. 4472908; Applied Biosystems; Thermo Fisher Scientific, Inc.) with $0.5 \mu 1$ cDNA on a CFX96 Real-Time system (Bio-Rad Laboratories, Inc., Hercules, CA, USA) according to the manufacturer's protocol. $\beta$-actin was used as an internal control to determine the levels of circATXN7 expression. The primer sequences used were as follows: $\beta$-actin forward, 5'-GAAATCGTGCGTGAC ATTAA-3', and reverse, 5'-AAGGAAGGCTGGAAGAGT G-3'; circATXN7 forward, 5'-CCTAGGGACAGAATTGGA CGA-3', and reverse, 5'-GCCCGCTCCGACATTCTT-3'. The qPCR reaction included an initial denaturation step in a 96-well optical plate at $95^{\circ} \mathrm{C}$ for $10 \mathrm{~min}$, followed by 40 cycles of $92^{\circ} \mathrm{C}$ for $15 \mathrm{sec}$ and $60^{\circ} \mathrm{C}$ for $1 \mathrm{~min}$. The relative levels of gene expression were normalized to $\beta$-actin and calculated using the $2^{-\Delta \Delta \mathrm{Cq}}$ method to determine fold changes of circATXN7 expression (tumor vs. normal) (17). The PCR products were subjected to Sanger sequencing by TSINGKE Biological Technology Co., Ltd. (Beijing, China).

Nucleic acid electrophoresis. The cDNA and gDNA PCR products were investigated using $4 \%$ agarose gel electrophoresis with TBE running buffer. The primer sequences used were as follows: divergent forward, 5'-CCTAGGGACAGA ATTGGACGA-3', and reverse, 5'-GCCCGCTCCGACATT CTT-3'; convergent forward, 5'-CTATCGTTTGCTGGGTTG CG-3', and reverse, 5'-ACTTTCGTCCAATTCTGTCCCT-3'.

Cell proliferation assay. Cell proliferation was detected using an MTT assay (Beijing Solarbio Science \& Technology Co., Ltd., Beijing, China) according to the manufacturer's protocol. Briefly, the transfected cells were seeded into 96-well plates ( 3,000 cells/well), and the proliferation rates were measured at $0,24,48,72$ and $96 \mathrm{~h}$ after transfection. A total of $20 \mu \mathrm{l}$ MTT solution was added to each well and incubated for $2 \mathrm{~h}$ at $37^{\circ} \mathrm{C}$. Next, the medium in each well was discarded, and $150 \mu \mathrm{l}$ dimethyl sulfoxide was added. Subsequent to shaking the plate for $15 \mathrm{~min}$, the absorbance was measured spectrophotometrically at $490 \mathrm{~nm}$. The data are representative of three individual experiments, where were conducted in triplicate.

For the EdU assays, at $24 \mathrm{~h}$ after transfection, $6 \times 10^{4}$ cells/well were seeded in a 24 -well plate. After a further $24 \mathrm{~h}$ of incubation, EdU was added to a final concentration of $50 \mu \mathrm{M}$ per well. Following incubation with EdU for $2 \mathrm{~h}$, the cells were fixed for $20 \mathrm{~min}$ with $150 \mu \mathrm{l} 4 \%$ formaldehyde solution in PBS. EdU labelling with the kFluor488-azide was performed using a kFluor488 Click-It EdU Cell Proliferation Assay kit (Nanjing KeyGen Biotech Co., Ltd., Nanjing, China). For excitation of kFluor488-azide, a 495-nm laser was used under a fluorescence microscope (Olympus Corporation, Tokyo, Japan).

Clonogenic assay. A total of 600 cells/well were transfected in a 6-well plate with $2 \mathrm{ml}$ medium containing $10 \%$ FBS. RPMI-1640 medium and DMEM were used for A549 and SPC-A1 cells, respectively. The medium was changed every 3 days. After 10 days, the cells were immobilized using $4 \%$ paraformaldehyde and stained with $0.1 \%$ crystal violet for $15 \mathrm{~min}$ at room temperature. The visible colonies of each well were then manually counted (11). 
A

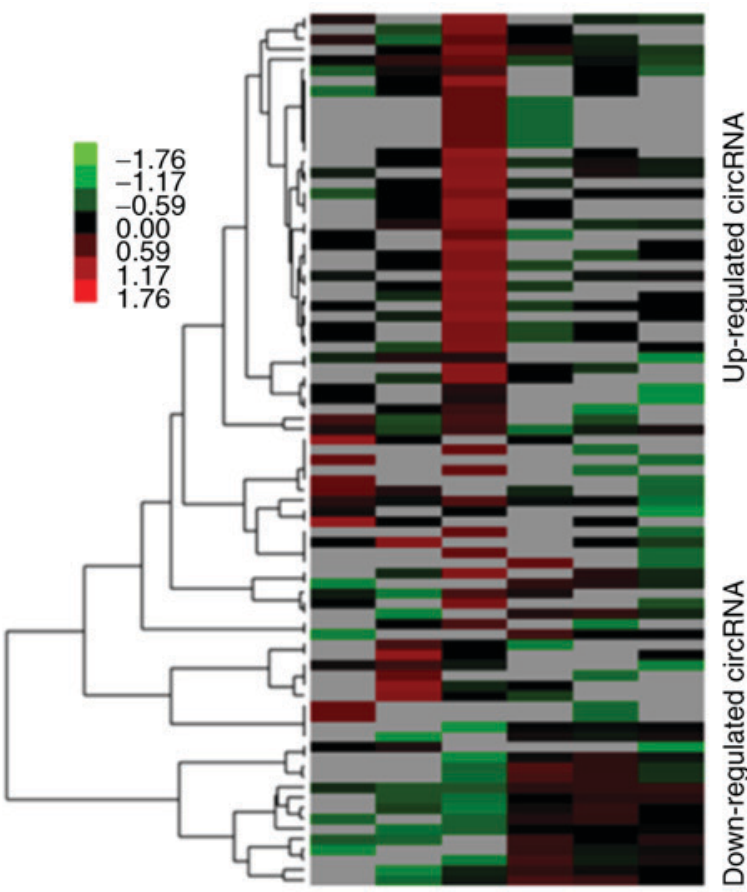

B

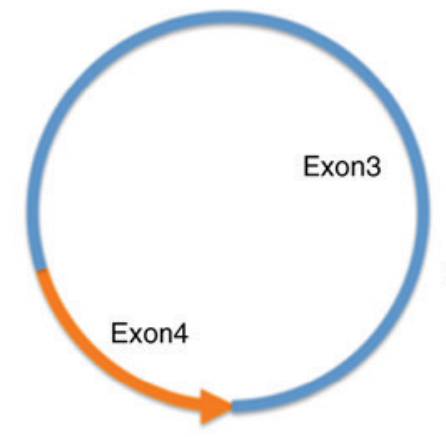

circATXN7
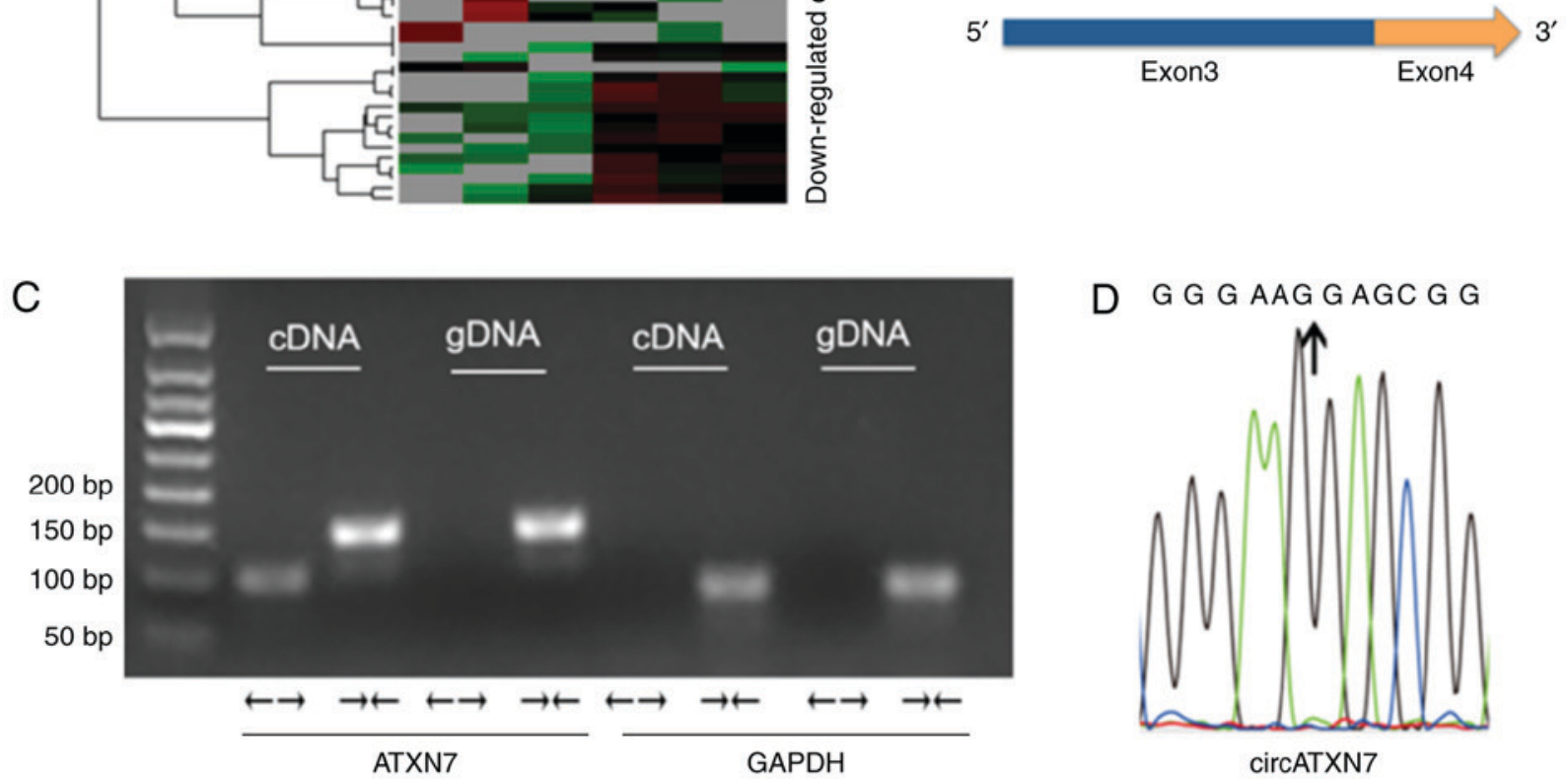

D G G G AAG G AGC G G

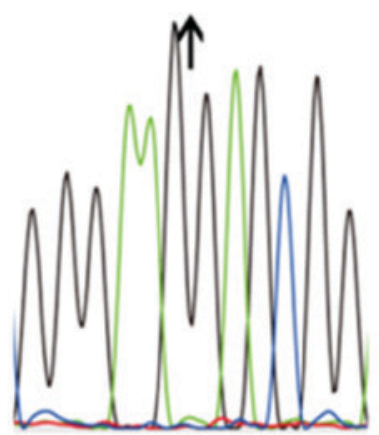

circATXN7

Figure 1. Expression profiles of circRNAs in NSCLC tissues compared with the adjacent normal tissues. (A) Heat map demonstrating the differential expression and hierarchical clustering of circRNAs between NSCLC and adjacent normal tissues. (B) circATXN7 is back-spliced by exons 3 and 4 of ATXN7. (C) Divergent primers detected circRNAs in cDNA, but not gDNA. The arrows indicate the direction of the primers: $\longleftrightarrow$ indicates divergent primers and $\rightarrow \leftarrow$ indicates convergent primers. (D) Sanger sequencing of cirATXN7 indicated the back-splice junction. The different colors represent different DNA bases. circRNA, circular RNA; NSCLC, non-small cell lung cancer.

Invasion assay. A Transwell assay was performed to investigate the migration and invasion of human lung cancer SPC-A1 and A549 cells. Briefly, the cells were transfected with $100 \mathrm{nM}$ siRNA-circATXN7 or siRNA-NC for $24 \mathrm{~h}$, and then plated at a density of $1 \times 10^{5}$ cells/well in the upper Transwell chamber (pore size, $8 \mu \mathrm{m}$; Corning, Inc., Corning, NY, USA), which contained $200 \mu \mathrm{l}$ of the corresponding serum-free medium. RPMI-1640 medium or DMEM containing 10\% FBS was added to the lower chamber for A549 and SPC-A1 cells, respectively. After $48 \mathrm{~h}$ of incubation at $37^{\circ} \mathrm{C}$ with $5 \% \mathrm{CO}_{2}$, the migrated cells on the filter surface were fixed with methanol and stained with $0.01 \%$ crystal violet for $15 \mathrm{~min}$ at room temperature, while the cells remaining in the upper membrane were discarded. The cell numbers were calculated in five random fields under a microscope (CTR6000; Leica Microsystems $\mathrm{GmbH}$, Wetzlar, Germany). Each experiment was performed in triplicate.
Statistical analysis. All statistical analyses in the present study were performed using the SPSS software package (version 20.0; IBM Corporation, Armonk, NY, USA) and GraphPad Prism software (version 7.0; GraphPad software Inc., La Jolla, CA, USA). All quantitative data are presented as the mean \pm standard deviation from at least three independent experiments. Two-tailed Student's t-test was used for the analysis of continuous variables. Differences among the three groups were analyzed by analysis of variance and Scheffe's test. The prognostic value of circATXN7 expression was further analyzed using the Kaplan-Meier method. $\mathrm{P}<0.05$ was considered to indicate a statistically significant difference.

\section{Results}

circRNAs in NSCLC. In our previous study, a number of circRNAs were detected to be overexpressed in NSCLC (15) 
Table I. Associations between circATXN7 level and clinicopathological characteristics of patients with non-small cell lung cancer.

\begin{tabular}{lccc}
\hline & \multicolumn{2}{c}{ circATNX7 } & \\
\cline { 2 - 3 } & $\begin{array}{c}\text { Low } \\
\text { expression } \\
(\mathrm{n}=12)\end{array}$ & $\begin{array}{c}\text { High } \\
\text { expression } \\
(\mathrm{n}=45)\end{array}$ & P-value \\
Characteristic & & & 0.577 \\
\hline Sex & 8 & 26 & \\
Male & 4 & 19 & \\
Female & & & 0.177 \\
Age, years & 9 & 24 & \\
$\geq 60$ & 3 & 21 & 0.058 \\
$<60$ & & & \\
TNM stage & 7 & 13 & 0.533 \\
I-II & 5 & 32 & \\
III-IV & & & \\
Lymph node metastasis & 6 & 18 & \\
No & 6 & 27 & \\
Yes & &
\end{tabular}

TMN, Tumor-Node-Metastasis; circATNX7, circular RNA ATNX7.

(Fig. 1A). In particular, one of the circRNAs was significantly overexpressed, which was subsequently defined as circATXN7, since it is derived from the ATXN7 gene. circATXN7 is back-spliced by exons 3 and 4 of ATXN7 (Fig. 1B). To characterize the circular form of circATXN7, convergent primers that amplify the linear transcript of ATXN7 and divergent primers that amplify circATXN7 were designed. The genomic DNA (gDNA) and complementary DNA (cDNA) of A549 cells were amplified by divergent and convergent primers, respectively. As indicated, the cDNA of the PCR products was amplified by the divergent primers, while both cDNA and gDNA of the PCR products were amplified by the convergent primers. GAPDH served as a negative control (Fig. 1C). Sanger sequencing confirmed the splicing junction site (Fig. 1D).

Aberrant expression of circATXN7 in NSCLC. To investigate the expression of circATXN7 in NSCLC, RT-qPCR analysis was performed in 57 pairs of NSCLC tissues and matched adjacent non-tumor tissues. It was observed that circATXN7 was upregulated in 45 of the 57 NSCLC tissues as compared with its expression in the matched non-tumor tissues (Fig. 2A). However, no significant associations were detected between the expression level of circATXN7 and the clinicopathological characteristics, including age, sex, lymph node metastasis or TNM stage (Table I) (18). The prognostic value of circATXN7 expression was demonstrated by the Kaplan-Meier survival curves. It was observed that the survival time of patients with high levels of circATXN7 was shorter compared with that of patients with low levels of circATXN7, although the association was not statistically significant ( $\mathrm{P}>0.05$; Fig. $2 \mathrm{~B})$.

circATXN7 promotes the proliferation and invasion abilities of NSCLC cells. SPC-A1 and A549 were selected as the
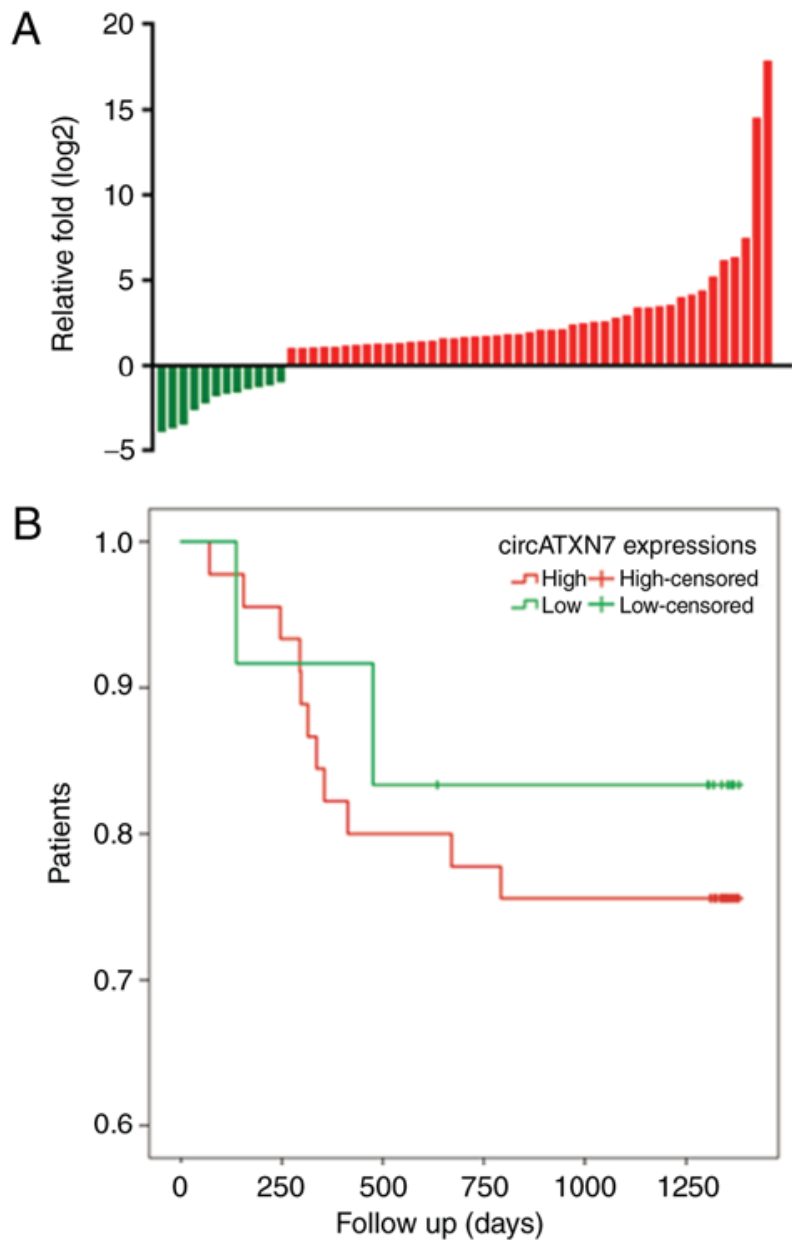

Figure 2. (A) circATXN7 expression was upregulated in 45 NSCLC tissues in comparison with the paired normal tissues, as determined by reverse transcription-quantitative polymerase chain reaction. (B) Correlation of NSCLC patient survival with the level of circATXN7 expression. NSCLC, non-small cell lung cancer.

experimental cell lines in the current study. Two siRNAs that specifically target the junction of the covalently joined $3^{\prime}$ and 5' ends were designed to inhibit circATXN7 expression and investigate its biological function in vitro, and the circATXN7 silencing in A549 and SPC-A1 cells was successfully induced by transfection (Fig. 3A). Next, the results of the MTT assay indicated that the silencing of circATXN7 significantly suppressed the proliferative ability compared with that in cells transfected with negative control (NC) siRNA (Fig. 3B). Furthermore, the clonogenic assay demonstrated that silencing circATXN7 significantly decreased the number of clones in NSCLC cells (Fig. 3C). The EdU assay also suggested that circATXN7 silencing inhibited the proliferative ability of A549 cells (Fig. 4). Thus, these results revealed that circATXN7 silencing suppressed the proliferation of NSCLC cells.

The effect of circATXN7 on the invasive ability of NSCLC cells was then further analyzed. The Transwell assay confirmed that the silencing of circATXN7 markedly inhibited the invasive ability of A549 and SPC-A1 cells (Fig. 5). Taken together, the results indicated that circATXN7 silencing was able to inhibit the proliferation and invasion of lung cancer cells in vitro. 

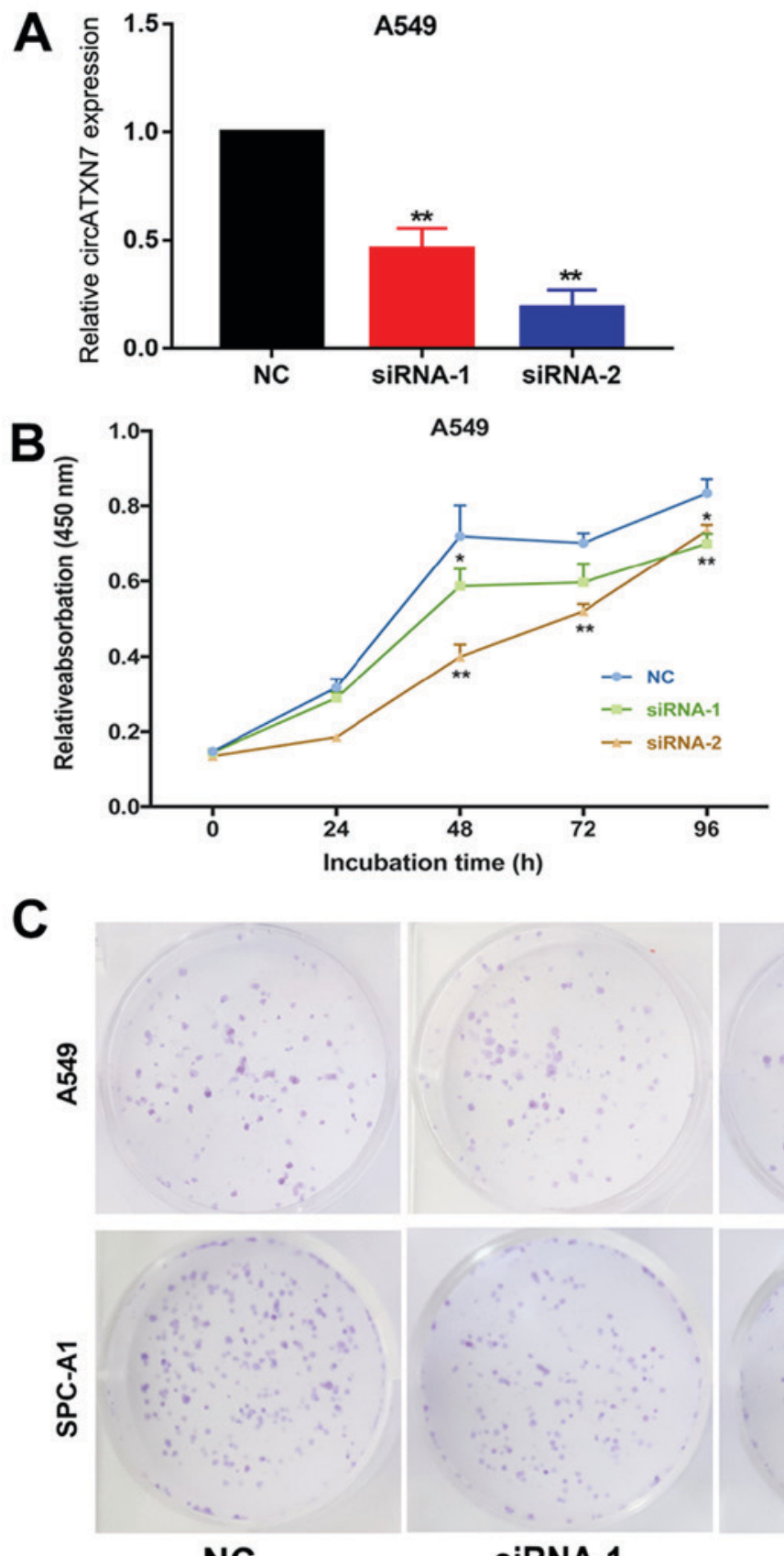

NC

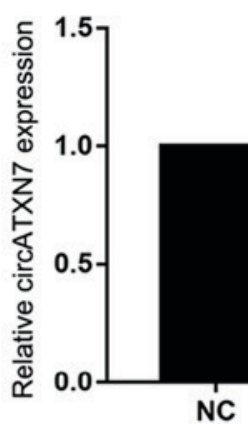

SPC-A1
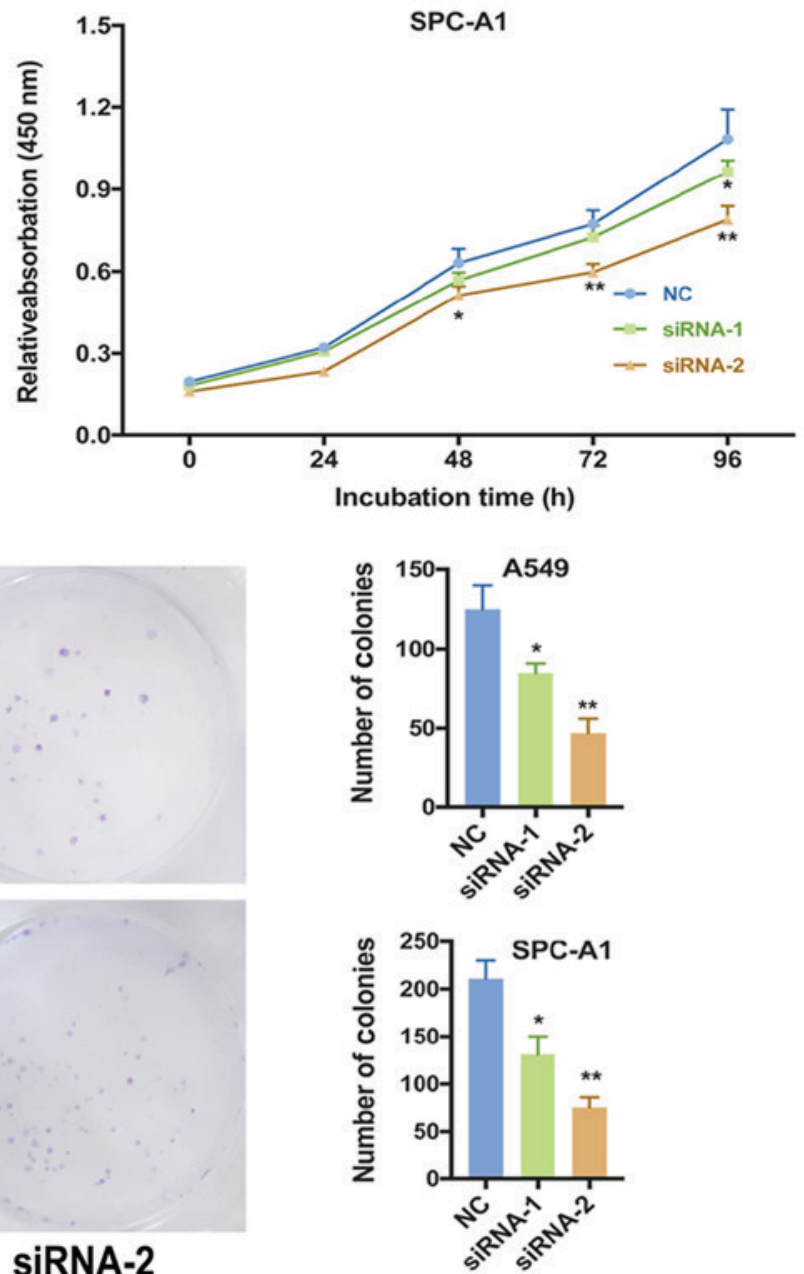

Figure 3. circATXN7 promotes the proliferation of two non-small cell lung cancer cell lines in vitro. (A) Reverse transcription-quantitative polymerase chain reaction analysis of circATXN7 following transfection with two siRNAs. (B) MTT assay indicates that siRNA transfection inhibited the proliferation of A549 cells and SPC-A1 cells. (C) Clonogenic assay, indicating the same results as the MTT assay. ${ }^{*} \mathrm{P}<0.05$ and ${ }^{* *} \mathrm{P}<0.01$ vs. NC group. siRNA, small interfering RNA; NC, negative control.

\section{Discussion}

circRNAs are a type of non-protein coding RNAs that are characterized by a covalently closed loop, and have been detected for decades in viruses, plants and animals (10). To date, the role and mechanism of circRNAs in the progression of malignancies remain to be identified (19). circRNAs are characterized by highly conserved sequences and have a specific covalently closed circular construction (20). They may serve considerable roles in the initiation and development of cancer, and may be candidate biomarkers for the diagnosis of diseases, such as cancer $(21,22)$.
The tumorigenesis of NSCLC is a complex dynamic biological process that involves multiple genes (23). However, a limited number of studies have examined the association between circRNAs and NSCLC. Previously, we demonstrated that a novel circular RNA, circPRKCI, functions as a sponge for both miR-545 and miR-589 in NSCLC and inhibits their suppression of the pro-tumorigenic transcription factor E2F7 (14).

In the present study, a novel circRNA, namely circATXN7, was identified in NSCLC. The potential association of the expression of circATXN7 with clinical factors and its prognostic value were investigated. Next, the potential function of 

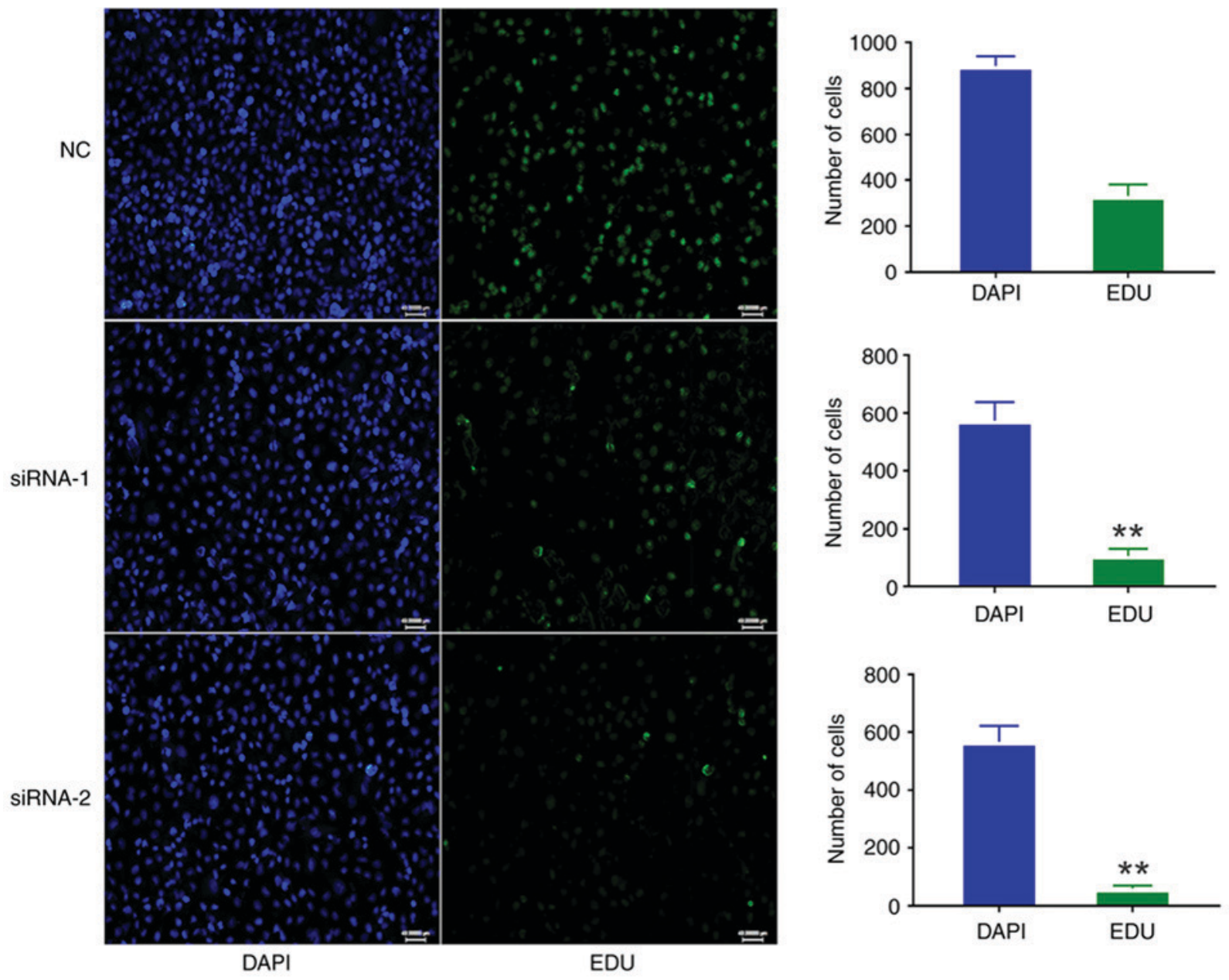

Figure 4. EDU assay demonstrated that transfection with siRNA targeting circATXN7 inhibited the proliferation of A549 cells. ${ }^{* *} \mathrm{P}<0.01$ vs. DAPI group. siRNA, small interfering RNA; NC, negative control.
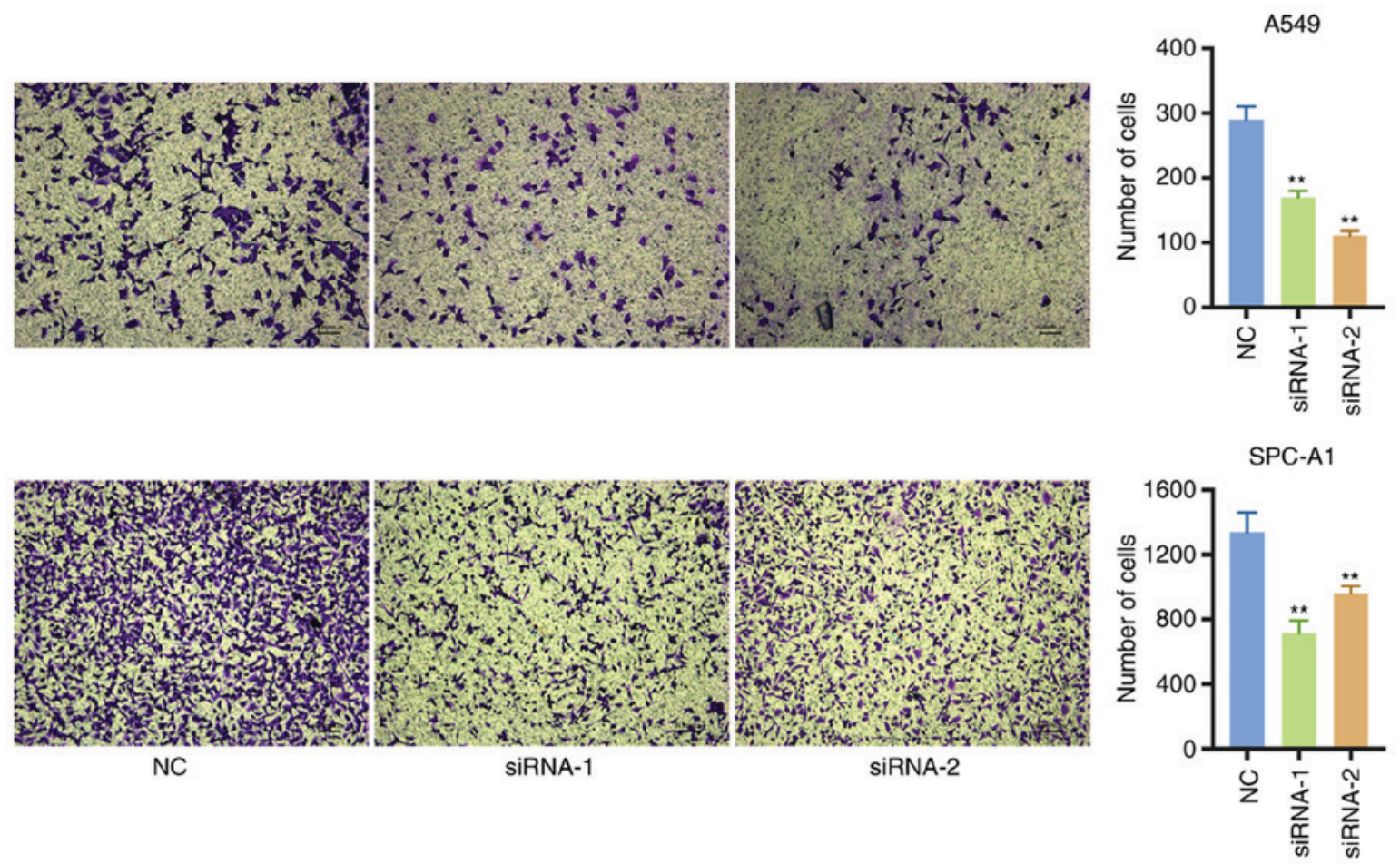

Figure 5. Transwell assay indicated that transfection with siRNA targeting circATXN7 inhibited the invasion ability of A549 and SPC-A1 cells. ${ }^{* *} \mathrm{P}<0.01$ vs. NC group. siRNA, small interfering RNA; NC, negative control. 
circATXN7 was investigated by siRNA-mediated silencing. The findings of the current study indicated that the prognosis of patients with upregulation of circATXN7 was poorer compared with patients with low circATXN7 levels; however, the association was not statistically significant. Furthermore, there was no marked association between circATXN7 expression level and the majority of clinicopathological characteristics, including the patient age, cancer location, lymph node metastasis, tumor size, tumor differentiation, $\mathrm{T}$ stage or TNM stage. Overall, the association between circATXN7 expression level and the overall survival of patients with NSCLC requires further research.

Silencing of circATXN7 by siRNA was subsequently conducted in the current study to investigate its biological functions in NSCLC cells. The results indicated that circATXN7 silencing inhibited the proliferation and invasion of NSCLC cells, suggesting the oncogenic role of circATXN7 in NSCLC. Hsu and Coca-Prados (24) detected circRNAs in eukaryote cells using electron microscopy in 1979, and circRNAs have since been hypothesized to be promising biomarkers in numerous diseases, particularly in cancer, owing to sequence conservation and biological stability $(25,26)$. circRNAs may harbor numerous miRNA binding sites, functioning as a huge 'sponges' that can consume target miRNAs (27). circHIPK3, which is derived from exon 2 of the HIPK3 gene, was reported to sponge 9 miRNAs with 18 potential binding sites (28). Therefore, further specific studies are required to analyze whether circATXN7 serves as a 'sponge' for miRNAs.

In conclusion, the present study is the first to identify that circATXN7 was upregulated in NSCLC tumor tissues. Furthermore, the downregulation of circATXN7 inhibited the proliferation and invasion abilities of NSCLC cells.

\section{Acknowledgements}

Not applicable.

\section{Funding}

The present study was supported by the National Natural Science Foundation of China (grant no. 81702256), the Natural Science Foundation of Beijing (grant no. 7182169) and partly by the Postdoctoral Fellowship of Peking-Tsinghua Center for Life Sciences (grant awarded to MQ; grant no. 4452-10148).

\section{Availability of data and materials}

The datasets used or analyzed during the current study are available from the corresponding author on reasonable request.

\section{Authors' contributions}

MQ, JW and QH designed experiments. QH, MQ, SW, XL, FY, $\mathrm{CF}$ and $\mathrm{KZ}$ performed the experiments. $\mathrm{MQ}$ and $\mathrm{QH}$ performed the data analysis and wrote the manuscript. All authors discussed the results and commented on the manuscript. All authors read and approved the final manuscript.

\section{Ethics approval and consent to participate}

All procedures in the current study involving human participants were performed in accordance with the standards of the Ethical Committee of Peking University People's Hospital, and with the 1964 Helsinki Declaration and its later amendments or comparable ethical standards. Informed consent was obtained from all individual participants included in the study.

\section{Patient consent for publication}

Not applicable.

\section{Competing interests}

The authors declare that they have no competing interests.

\section{References}

1. Smith RA, Manassaram-Baptiste D, Brooks D, Doroshenk M, Fedewa S, Saslow D, Brawley OW and Wender R: Cancer screening in the United States, 2015: A review of current American cancer society guidelines and current issues in cancer screening. CA Cancer J Clin 65: 30-54, 2015.

2. Ettinger DS, Wood DE, Akerley W, Bazhenova LA, Borghaei H, Camidge DR, Cheney RT, Chirieac LR, D'Amico TA, Demmy TL, et al: Non-small cell lung cancer, version 6.2015. J Natl Compr Canc Netw 13: 515-524, 2015.

3. Travis WD, Brambilla E, Nicholson AG, Yatabe Y, Austin JHM, Beasley MB, Chirieac LR, Dacic S, Duhig E, Flieder DB, et al: The 2015 World health organization classification of lung tumors: Impact of genetic, clinical and radiologic advances since the 2004 classification. J Thorac Oncol 10: 1243-1260, 2015.

4. Antoni D and Mornex F: Chemoradiotherapy of locally advanced nonsmall cell lung cancer: State of the art and perspectives. Curr Opin Oncol 28: 104-109, 2016.

5. Socinski MA, Obasaju C, Gandara D, Hirsch FR, Bonomi P, Bunn P, Kim ES, Langer CJ, Natale RB, Novello S, et al: Clinicopathologic features of advanced squamous NSCLC. J Thorac Oncol 11: 1411-1422, 2016.

6. Zhu X, Wang X, Wei S, Chen Y, Chen Y, Fan X, Han S and Wu G: hsa_circ_0013958: A circular RNA and potential novel biomarker for lung adenocarcinoma. FEBS J 284: 2170-2182, 2017.

7. Lasda E and Parker R: Circular RNAs: Diversity of form and function. RNA 20: 1829-1842, 2014.

8. Hentze MW and Preiss T: Circular RNAs: Splicing's enigma variations. EMBO J 32: 923-925, 2013.

9. Salmena L, Poliseno L, Tay Y, Kats L and Pandolfi PP: A ceRNA hypothesis: The rosetta stone of a hidden RNA language? Cell 146: 353-358, 2011

10. Xu Y, Wang J, Qiu M, Xu L, Li M, Jiang F, Yin R and Xu L: Upregulation of the long noncoding RNA TUG1 promotes proliferation and migration of esophageal squamous cell carcinoma. Tumour Biol 36: 1643-1651, 2015.

11. Chen I, Chen CY and Chuang TJ: Biogenesis, identification, and function of exonic circular RNAs. Wiley Interdiscip Rev RNA 6: 563-579, 2015.

12. Xie H, Ren X, Xin S, Lan X, Lu G, Lin Y, Yang S, Zeng Z, Liao W, Ding YQ and Liang L: Emerging roles of circRNA_001569 targeting miR-145 in the proliferation and invasion of colorectal cancer. Oncotarget 7: 26680-26691, 2016.

13. Zhong Z, Lv M and Chen J: Screening differential circular RNA expression profiles reveals the regulatory role of circTCF25-miR-103a-3p/miR-107-CDK6 pathway in bladder carcinoma. Sci Rep 6: 30919, 2016.

14. Qiu M, Xia W, Chen R, Wang S, Xu Y, Ma Z, Xu W, Zhang E, Wang J, Fang T, et al: The circular RNA circPRKCI promotes tumor growth in lung adenocarcinoma. Cancer Res 78: 2839-2851, 2018.

15. Ding X, Zhang S, Li X, Feng C, Huang Q, Wang S, Wang S, Xia W, Yang F, Yin R, et al: Profiling expression of coding genes, long noncoding RNA, and circular RNA in lung adenocarcinoma by ribosomal RNA-depleted RNA sequencing. FEBS Open Bio 8: 544-555, 2018. 
16. Detterbeck FC, Boffa DJ, Kim AW and Tanoue LT: The eighth edition lung cancer stage classification. Chest 151: 193-203, 2017.

17. Huang SD, Yuan Y, Zhuang CW, Li BL, Gong DJ, Wang SG, Zeng ZY and Cheng HZ: MicroRNA-98 and microRNA-214 post-transcriptionally regulate enhancer of zeste homolog 2 and inhibit migration and invasion in human esophageal squamous cell carcinoma. Mol Cancer 11: 51, 2012.

18. Goldstraw P, Chansky K, Crowley J, Rami-Porta R, Asamura H, Eberhardt WE, Nicholson AG, Groome P, Mitchell A, Bolejack V, et al: The IASLC lung cancer staging project: Proposals for revision of the TNM stage groupings in the forthcoming (Eighth) edition of the TNM classification for lung cancer. J Thorac Oncol 11: 39-51, 2016.

19. Wang X, Sun Q, Chen C, Yin R, Huang X, Wang X, Shi R, Xu L and Ren B: ZYG11A serves as an oncogene in non-small cell lung cancer and influences CCNE1 expression. Oncotarget 7: 8029-8042, 2016.

20. Vannini I, Wise PM, Challagundla KB, Plousiou M, Raffini M, Bandini E, Fanini F, Paliaga G, Crawford M, Ferracin M, et al: Transcribed ultraconserved region 339 promotes carcinogenesis by modulating tumor suppressor microRNAs. Nat Commun 8: $1801,2017$.

21. Hansen TB, Jensen TI, Clausen BH, Bramsen JB, Finsen B, Damgaard CK and Kjems J: Natural RNA circles function as efficient microRNA sponges. Nature 495: 384-388, 2013.
22. Ebbesen KK, Hansen TB and Kjems J: Insights into circular RNA biology. RNA Biol 14: 1035-1045, 2017.

23. Chen Y, Li C, Tan C and Liu X: Circular RNAs: A new frontier in the study of human diseases. J Med Genet 53: 359-365, 2016.

24. Hsu MT and Coca-Prados M: Electron microscopic evidence for the circular form of RNA in the cytoplasm of eukaryotic cells. Nature 280: 339-340, 1979.

25. Salzman J, Gawad C, Wang PL, Lacayo N and Brown PO: Circular RNAs are the predominant transcript isoform from hundreds of human genes in diverse cell types. PLoS One 7: e30733, 2012.

26. Li P, Chen S, Chen H, Mo X, Li T, Shao Y, Xiao B and Guo J: Using circular RNA as a novel type of biomarker in the screening of gastric cancer. Clin Chim Acta 444: 132-136, 2015.

27. Zhao ZJ and Shen J: Circular RNA participates in the carcinogenesis and the malignant behavior of cancer. RNA Biol 14: 514-521, 2017.

28. Zheng Q, Bao C, Guo W, Li S, Chen J, Chen B, Luo Y, Lyu D, Li Y, Shi G, et al: Circular RNA profiling reveals an abundant circHIPK 3 that regulates cell growth by sponging multiple miRNAs. Nat Commun 7: 11215, 2016.

This work is licensed under a Creative Commons Attribution-NonCommercial-NoDerivatives 4.0 International (CC BY-NC-ND 4.0) License. 